\title{
Social and emotional relevance in face processing: happy faces of future interaction partners enhance the late positive potential
}

\author{
Florian Bublatzky*, Antje B. M. Gerdes, Andrew J. White, Martin Riemer and Georg W. Alpers
}

School of Social Sciences, Clinical Psychology, Biological Psychology, and Psychotherapy, University of Mannheim, Mannheim, Germany

\section{Edited by:}

Aina Puce, Indiana University, USA

Reviewed by:

Gilles Pourtois, University of Ghent, Belgium

Elizabeth Bendycki DaSilva, Indiana University, USA

\section{*Correspondence:}

Florian Bublatzky, School of Social Sciences, Clinical Psychology,

Biological Psychology, and

Psychotherapy, University of

Mannheim, L13, 17, 68131

Mannheim, Germany

e-mail:f.bublatzky@

uni-mannheim.de

\begin{abstract}
Human face perception is modulated by both emotional valence and social relevance, but their interaction has rarely been examined. Event-related brain potentials (ERP) to happy, neutral, and angry facial expressions with different degrees of social relevance were recorded. To implement a social anticipation task, relevance was manipulated by presenting faces of two specific actors as future interaction partners (socially relevant), whereas two other face actors remained non-relevant. In a further control task all stimuli were presented without specific relevance instructions (passive viewing). Face stimuli of four actors ( 2 women, from the KDEF) were randomly presented for $1 \mathrm{~s}$ to 26 participants (16 female). Results showed an augmented N170, early posterior negativity (EPN), and late positive potential (LPP) for emotional in contrast to neutral facial expressions. Of particular interest, face processing varied as a function of experimental tasks. Whereas task effects were observed for P1 and EPN regardless of instructed relevance, LPP amplitudes were modulated by emotional facial expression and relevance manipulation. The LPP was specifically enhanced for happy facial expressions of the anticipated future interaction partners. This underscores that social relevance can impact face processing already at an early stage of visual processing. These findings are discussed within the framework of motivated attention and face processing theories.
\end{abstract}

Keywords: face processing, social interaction, emotion, anticipation, ERP

\section{INTRODUCTION}

Humans are intrinsically social. From an evolutionary perspective, social information is critical for survival as it contributes to successful commitment, procreation and preservation (Tooby and Cosmides, 1992; Brothers, 2002). Thus, conspecifics are primary elicitors of emotions designed to promote both affiliation and protection in the face of constantly changing environmental conditions (Keltner and Kring, 1998). Accordingly, viewing facial stimuli is highly informative and mediates perceptual, physiological, and behavioral responses (Hamm et al., 2003; Vuilleumier and Pourtois, 2007).

To investigate the link between social and emotional information processing, the present study focuses on the social relevance of facial pictures. Human faces contain salient social signals mediating information about one's own and the others' identity, emotional state, and intentions (Ekman and Friesen, 1975; Öhman, 1986). The neural signature of face processing has been outlined in recent research (Haxby et al., 2002; Adolphs and Spezio, 2006). Given the crucial importance of being able to efficiently read and understand facial expressions, it has been proposed that distinct brain structures are centrally involved in face processing (e.g., fusiform face area (FFA), superior temporal sulcus (STS); Kanwisher et al., 1997 but see Chao et al., 1999). In addition, research has identified several neural substrates involved in both emotional and social processes (e.g., amygdala, insular, medial prefrontal cortex (MPFC); Gusnard et al., 2001; Norris et al., 2004; Williams et al., 2004; Northoff et al., 2006; Schmitz and Johnson, 2007; Olsson and Ochsner, 2008; Sabatinelli et al., 2011). In order to adequately interact in social situations, observing emotional facial expressions facilitates perceptual, attentional, and behavioral responses (Alpers and Gerdes, 2007; Alpers et al., 2011). For instance, in visual search tasks, threatening (schematic) faces are detected more quickly than friendly or neutral target faces especially among highly anxious participants (Byrne and Eysenck, 1995; Öhman et al., 2001). In line with an evolutionary perspective, this processing advantage has been described specifically for angry and fearful faces mediating potential threat to the observer (Byrne and Eysenck, 1995; Whalen et al., 2001).

Electrophysiological measures are particularly well-suited to investigate the temporal dynamics of face processing. Eventrelated brain potential (ERP) studies have revealed processing differences for facial stimuli within the first $100 \mathrm{~ms}$ after stimulus onset. For instance, suggested to reflect attention gain control in extrastriate sources, enhanced P1 amplitudes were observed for fearful compared to neutral faces in visuo-spatial attention tasks (Pourtois et al., 2004). Further, temporo-occipital negativities have been shown to be sensitive to facial stimuli (N170; Bentin et al., 1996) and emotional facial expression (early posterior 
negativity, EPN; Schupp et al., 2004). The N170 is probably the most frequently investigated ERP component in face processing. It has been primarily related to the structural encoding of faces in temporo-occipital processing areas; for instance, as evidenced by studies manipulating structural features (e.g., face inversion; Itier and Taylor, 2002), presentation of specific face and body parts (e.g., only eye region; Itier et al., 2006), and spatial attention tasks (Holmes et al., 2003; Jacques and Rossion, 2007). Regarding the emotional state and intentions conveyed by facial expression, an early posterior negativity (occipito-temporal EPN; 150-300 ms) and late positive potentials (centro-parietal LPP; 300-700 ms) have been observed for angry as compared to neutral faces, but also for happy faces (Sato et al., 2001; Liddell et al., 2004; Schupp et al., 2004; Williams et al., 2006; Holmes et al., 2008; but see Wangelin et al., 2012). Further, these effects were more pronounced in socially anxious participants (Moser et al., 2008; Sewell et al., 2008; but see Mühlberger et al., 2009) and participants undergoing socially-mediated aversive anticipation (Wieser et al., 2010; Bublatzky and Schupp, 2012). Of particular interest, enhanced LPP amplitudes have been observed for neutral expressions of primed familiar faces (Schweinberger et al., 2002; Kaufmann et al., 2008), and when faces are high in social relevance (e.g., romantic partner, family members; Guerra et al., 2012).

The effects of emotional stimulus content on attention have also been documented with a variety of other visual stimuli (e.g., pictures of naturalistic scenes, words, and hand gestures; Schupp et al., 2006a; Kissler et al., 2007; Flaisch et al., 2009; Schacht and Sommer, 2009). Further, EPN and LPP components were found to vary as a function of emotional arousal (i.e., pronounced EPN/LPP for highly emotional arousing pictures; Schupp et al., 2006a), and the LPP appeared sensitive to emotion regulation (Hajcak et al., 2010; Thiruchselvam et al., 2011). In addition, both ERP components have been observed to occur spontaneously while passive picture viewing and during performance of concurrent explicit attention tasks (Schupp et al., 2006a; Pourtois et al., 2013). These results are in line with those of several neuroimaging studies (i.e., showing increased BOLD responses in distributed occipital, parietal and inferior temporal networks; Junghöfer et al., 2006; Sabatinelli et al., 2011) and studies that have shown clear differences in autonomic and reflex activity for emotional compared to neutral stimuli (e.g., Bradley et al., 2001). In sum, there is ample evidence supporting the notion that EPN and LPP components reflect motivationally guided selective attention to significant stimuli (Schupp et al., 2006a).

Building on these findings, the present study examined the joint effects of social relevance of facial stimuli and the displayed emotional expressions. Using an instructional learning paradigm, participants were informed that they would later be introduced to the person presented in a specific face picture. Thus, these faces acquired social relevance by virtue of being potential interaction partners and were contrasted with other non-relevant face actors. Furthermore, the manipulation of facial expressions (happy, neutral, angry) allowed to model the emotional valence and arousal of the anticipated social situation. In light of previous research on face processing, electrocortical processing was hypothesized to differentiate social relevant from non-relevant faces (enhanced EPN/LPP). Further, valence effects are proposed to account for prioritized emotion processing (e.g., threat- or happy-advantage; Schupp et al., 2004; Williams et al., 2006). For instance, based on higher motivational impact, emotional compared to neutral face processing may benefit from additional social relevance as reflected by enhanced LPP amplitudes (Schupp et al., 2007). Integrating different experimental paradigms and methodologies, the present study constitutes a new experimental approach to examine the mutual impact of social and emotional processes by means of the anticipation of a socially relevant situation.

\section{METHODS \\ PARTICIPANTS}

The sample consisted of 26 healthy volunteers ( 16 females) aged between 19 and 34 years $(M=23, S D=4.3)$ and recruited from the University of Mannheim (STAI-State $M=35.3, S D=4.6$; STAI-Trait $M=38.8, S D=7.7$; SIAS $M=16, S D=7.4$; FNEbrief version $M=33.9, S D=7.8$ ). All participants were informed about the study protocol before providing informed consent in accordance with the university's ethics guidelines. Participants received course credits for their participation.

\section{MATERIALS AND PRESENTATION}

Happy, neutral, and angry facial expressions of 4 different face actors (2 female) were selected from the Karolinska Directed Emotional Faces (KDEF; Lundqvist et al., 1998). ${ }^{1}$ Pictures (1024 $\times 768$ pixels) were randomly presented for $1 \mathrm{~s}$ without interstimulus gaps (see Figure 1). The full set of pictures $(N=12)$ was presented 60 times during two separate blocks, each consisting of 720 trials. The first block served as a control condition without specific instructions (passive viewing task). For the second block (meet task), two specific face actors (1 female and 1 male) were introduced as future interaction partners. Accordingly, two face actors were instructed as relevant whereas the other two face actors were non-relevant with respect to future interaction. Assignment of face stimuli to the relevant/non-relevant condition was counterbalanced across participants. Within blocks, face pictures were presented in a different order for each participant. Accounting for potential repetition effects (see Flaisch et al., 2008), picture randomization was restricted to no more than three repetitions of the same facial expression, equal transition probabilities between facial expressions and face actors, and no immediate repetition of the same actor displaying the same facial expression. Pictures were presented on a 22 inch computer screen located approximately 1 meter in front of the participants.

\section{PROCEDURE}

After the EEG sensor net was attached, participants were seated in a dimly-lit and sound-attenuated room. During a practice run (12 picture trials), participants were familiarized with the picture viewing procedure. In the following passive viewing task participants were instructed to attend to each picture appearing on the screen. Before the meet task, instructions were given concerning the relevance of face stimuli by indicating who were

${ }^{1}$ KDEF identifiers: actor 1: af20has, af20nes, af20ans; actor 2: af25has, af25nes, af25ans; actor 3: am10has, am10nes, am10ans; actor 4: am25has, am25nes, am25ans. 


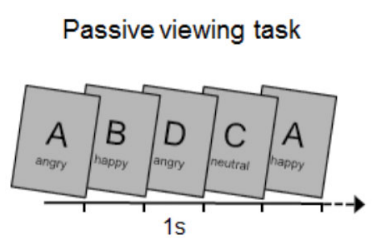

FIGURE 1 | Illustration of the experimental procedure. Pictures of 4 face actors (A, B, C, D) displaying happy, neutral, and angry facial expressions were presented randomly ( $1 \mathrm{~s}$ each) in 2 experimental tasks (each 720 trials) Participants were instructed to attend all stimuli in the passive viewing task.

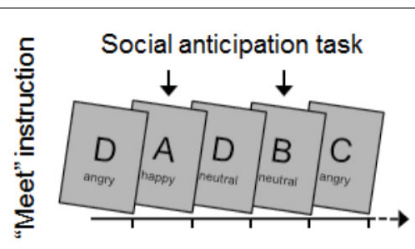

Following picture ratings, the main instruction about social contingencies was given: "You are going to meet one of these two people at the end of the experiment" (here indicated by an arrow). Finally, participants again rated face stimuli and were debriefed. the relevant and non-relevant face actors. With respect to the kind of interaction situation, the meet instruction was deliberately kept vague and neutral ("You are going to meet one of these two people at the end of the experiment"). After each block, valence and arousal ratings of the picture set was assessed using the paperpencil version of the self-assessment manikin (SAM; Bradley and Lang, 1994). At the end of the experiment, a debriefing interview was completed.

\section{EEG RECORDING}

Electrophysiological data were collected using a 64 actiCap system (BrainProducts, Munich, Germany) with $\mathrm{Ag} / \mathrm{AgCl}$ active electrodes mounted into a cap according to the 10-10 system (Falk Minow Services, Herrsching, Germany). The EEG was recorded continuously with a sampling rate of $500 \mathrm{~Hz}$ with $\mathrm{FCz}$ as the reference electrode, and filtered on-line from $0.1-100$ $\mathrm{Hz}$ using VisionRecorder acquisition software and BrainAmp DC amplifiers (BrainProducts, Munich, Germany). Impedances were kept below $10 \mathrm{k} \Omega$. Off-line analyses were performed using VisionAnalyzer 2.0 (BrainProducts) and EMEGS (Peyk et al., 2011) and included low-pass filtering at $30 \mathrm{~Hz}$, artifact detection, sensor interpolation, baseline-correction, and conversion to an average reference (Junghöfer et al., 2000). Stimulus-synchronized epochs were extracted and lasted from $100 \mathrm{~ms}$ before to $800 \mathrm{~ms}$ after stimulus onset. Finally, separate average waveforms were calculated for the experimental conditions Facial Expression (happy, neutral, angry), Task (passive, meet), and Relevance (relevant, non-relevant), ${ }^{2}$ for each sensor and participant.

\section{DATA REDUCTION AND ANALYSES \\ Self-report data}

Valence and arousal ratings were analyzed with repeated measures ANOVAs including the factors Facial Expression (happy, neutral, angry), Task (passive, meet), and Relevance (relevant, non-relevant).

\section{Event-related potentials}

To examine the effects of facial expression, instructed task, and relevance on face processing, a two-step procedure was used. As a first step, visual inspection and single sensor waveform analysis were used in concert to identify relevant ERP components. To

\footnotetext{
${ }^{2}$ Note: As the passive viewing task did not contain a relevance manipulation, an artificial data split was undertaken to adjust factor structure (i.e., each 1 male and 1 female face actor were assigned artificially to relevant/non-relevant condition).
}

this end, single sensor waveform analyses were calculated for each time point and each sensor separately (see Peyk et al., 2011) for the factors Facial Expression (happy, neutral, angry), Task (passive, meet), and Relevance (relevant, non-relevant). To correct for multiple testing, effects were only considered meaningful when the effects were observed for at least eight continuous data points and two neighboring sensors (cf., Bublatzky and Schupp, 2012). Supporting this cluster selection procedure, visual inspection helped ensure that no effects relevant to the main hypothesis regarding the interaction between Facial Expression, Task, and Relevance were missed.

Following this, conventional ERP analyses were based on area scores. Repeated measures ANOVAs based on mean activity in selected sensor clusters and time windows were performed. The P1 component was scored over parieto-occipital cluster (left: O1, PO3; right: O2, PO4) within 100 and $140 \mathrm{~ms}$ after picture onset. The N170 was scored at P7 and P8 between 150 and 200 ms. The EPN component was scored at bilateral posterior sensors (PO9 and PO10) between 260 and $360 \mathrm{~ms}$ after stimulus onset. To account for the broad distribution of the LPP component, mean activity was scored in bilateral centro-parietal clusters (left: FC1, $\mathrm{C} 1, \mathrm{CP} 1, \mathrm{P} 1$; right: $\mathrm{FC} 2, \mathrm{C} 2, \mathrm{CP} 2, \mathrm{P} 2)$ in a time window from 450-700 ms.

An overall multivariate ANOVA tested interaction effects between Facial Expression (happy, neutral, angry), Task (passive, meet), Relevance (relevant, non-relevant), and Laterality (left, right) as a function of ERP Component (P1, N170, EPN, LPP) using Wilks statistics. Significant main effects were observed for Component, $F_{(3,23)}=30.72, p<0.001, \eta_{p}^{2}=0.80$, Facial Expression, $F_{(2,24)}=11.78, p<0.001, \eta_{p}^{2}=0.50$, Task, $F_{(1,25)}=8.51, p<0.01, \eta_{p}^{2}=0.25$, but not for Relevance, $F_{(1,25)}=0.15, p=0.70, \eta_{p}^{2}=0.01$, or Laterality, $F_{(1,25)}=3.35$, $p=0.08, \eta_{p}^{2}=0.12$. Of particular importance, higher-order interactions were revealed for Component by Facial Expression, $F_{(6,20)}=9.81, p<0.001, \eta_{p}^{2}=0.75$, and Component by Task, $F_{(3,23)}=16.69, p<0.001, \eta_{p}^{2}=0.69$. Directly testing the interaction between the three task-sensitive ERP components (P1, EPN, LPP) revealed significant variation of Component as a function of Facial Expression, $F_{(4,22)}=13.23$, $p<0.001, \eta_{p}^{2}=0.71$, and Task, $F_{(2,24)}=26.13, p<0.001$, $\eta_{p}^{2}=0.69$. To follow up on these interactions, separate repeated measures ANOVAs including the factors Facial Expression, Task, Relevance, and Laterality were conducted for each ERP component. 
For effects involving repeated measures, the GreenhouseGeisser procedure was used to correct for violations of sphericity, and as a measure of effect size the partial $\eta^{2}\left(\eta_{p}^{2}\right)$ are reported. To control for type 1 error, Bonferroni correction was applied for post hoc $t$-tests.

\section{RESULTS}

\section{SELF-REPORT DATA}

Overall, valence ratings differed significantly for Facial Expression, $F_{(2,48)}=308.06, p<0.001, \eta_{p}^{2}=0.93$. Happy facial expressions $(M=7.86, S D=0.16)$ were rated more pleasant than neutral and angry faces $(M=5.15$ and $2.44, S D=0.16$ and 0.14$), p s<0.001$, and neutral as more pleasant than angry faces, $p<0.001$. Although a marginal significant main effect of Task, $F_{(1,24)}=3.78, p=0.06, \eta_{p}^{2}=0.14$, indicated that faces were rated as more pleasant during meet task, the interaction Facial Expression by Task was not significant, $F_{(2,48)}=1.34, p=0.27$, $\eta_{p}^{2}=0.05$. Neither instructed Relevance, $F_{(1,24)}=0.57, p=0.27$, $\eta_{p}^{2}=0.05$, nor any higher-order interaction reached significance, Fs $<1$, ps $>0.70, \eta_{p}^{2}<0.01$.

Arousal ratings varied for Facial Expression, $F_{(2,48)}=45.82$, $p<0.001, \eta_{p}^{2}=0.66$. Both happy and angry facial expressions $(M=4.21$ and $5.81, S D=0.38$ and 0.33$)$ were rated as more arousing than neutral $(M=2.62, S D=0.29)$, $p s<0.001$, and angry faces as more arousing than happy expressions, $p<0.01$. No main effects were observed for Task or Relevance, $F s_{(1,24)}=$ 2.79 and $1.67, p s=0.11$ and $0.21, \eta_{p}^{2}=0.10$ and 0.07 . However, arousal ratings varied as a function of Facial Expression by Task, $F_{(2,48)}=6.28, p<0.01, \eta_{p}^{2}=0.21$. To follow up on the differential impact of passive viewing and meet task, facial expressions were tested separately.

Happy face pictures, were rated as more arousing during passive viewing than meet task, Task $F_{(1,24)}=14.32, p<0.01, \eta_{p}^{2}=$ 0.37. Neither Relevance, $F_{(1,24)}=0.01, p=0.93, \eta_{p}^{2}<0.01$, nor the interaction Task by Relevance reached significance, $F_{(1,24)}=1.08$, $p=0.31, \eta_{p}^{2}=0.04$. Similarly, angry faces were rated higher in arousal during passive viewing compared to meet task, $F_{(1,24)}=$ 7.48, $p<0.05, \eta_{p}^{2}=0.24$. Neither Relevance, $F_{(1,24)}=3.09, p=$ $0.09, \eta_{p}^{2}=0.11$, nor Task by Relevance, $F_{(1,24)}=0.13, p=0.72$, $\eta_{p}^{2}<0.01$, reached significance. In contrast, arousal ratings for neutral faces did not vary by Task, $F_{(1,24)}=1.0, p=0.33, \eta_{p}^{2}=0.04$, Relevance, $F_{(1,24)}=0.12, p=0.73, \eta_{p}^{2}=0.01$, or Task by Relevance, $F_{(1,24)}<0.01, p=1.0, \eta_{p}^{2}<0.01$.

\section{EVENT-RELATED POTENTIALS}

Results indicated that verbal instructions about future interaction partners modulated early and late face processing as revealed by enhanced P1, EPN, and LPP amplitudes (Figures 2, 3). Further, the interaction of social and emotional relevance varied across the visual processing stream. Whereas early components revealed independent main effects of Facial Expression and Task (shown by P1, N170, and EPN), the LPP was markedly augmented for happy faces considered as future interaction partners (Figure 4).

\section{P1 component}

Enhanced P1 amplitude for the meet compared to passive viewing task reached marginal significance, $F_{(1,25)}=3.57, p=0.07$, $\eta_{p}^{2}=0.13$, however, instructed Relevance did not increase P1 amplitude, $F_{(1,25)}=0.01, p=0.92, \eta_{p}^{2}<0.01$. Further, emotional Facial Expression modulated the P1 component, $F_{(2,50)}=7.44$, $p<0.01, \eta_{p}^{2}=0.23$. Follow-up tests revealed that amplitudes were more pronounced for angry facial expressions compared to neutral and happy faces, $F \mathrm{~s}_{(1,25)}=8.71$ and 10.72 , $p \mathrm{~s}<0.01$, $\eta_{p}^{2}=0.26$ and 0.30 . The difference between happy and neutral facial expressions was not statistically significant, $F_{(1,25)}=0.70$, $p=0.41, \eta_{p}^{2}=0.03$. The P1 amplitude was more pronounced over the right hemisphere, $F_{(1,25)}=8.79, p<0.01, \eta_{p}^{2}=0.26$. No further interactions including Facial Expression, Task or Relevance reached statistical significance, $F s<1.76$, ps $>0.18$, $\eta_{p}^{2}<0.07$

\section{N170 component}

Whereas Task and Relevance did not modulate the N170, $F_{\mathrm{s}_{(1,25)}}=0.31$ and 0.33, $p=0.58$ and 0.57, $\eta_{p}^{2}=0.01$ and 0.01 , amplitudes varied as a function of Facial Expression, $F_{(2,50)}=9.23, p=0.001, \eta_{p}^{2}=0.27$. The N170 was more pronounced for both happy and angry faces compared to neutral facial expressions, $F s_{(1,25)}=26.70$ and 3.98, $p$ s $<0.001$ and $=0.06, \eta_{p}^{2}=0.52$ and 0.14 . The difference between happy and angry faces reached marginal significance, $F_{(1,25)}=$ 4.02, $p=0.06, \eta_{p}^{2}=0.14$. No main effect of Laterality was observed, $F_{(1,25)}=2.75, p=0.11, \eta_{p}^{2}=0.10$, nor any interaction including Facial Expression, Task, and Relevance reached statistical significance, $F s<0.71, p s>0.48$, $\eta_{p}^{2}<0.03$

\section{Early posterior negativity}

More pronounced negativity was observed for the meet compared to the passive viewing task, $F_{(1,25)}=43.61$, $p<0.001, \eta_{p}^{2}=0.64$, however, relevance instruction did not modulate the EPN, $F_{(1,25)}=0.80, p=0.38, \eta_{p}^{2}=0.03$. Replicating previous findings, the EPN amplitude varied as a function of Facial Expression, $F_{(2,50)}=16.28, p<0.001, \eta_{p}^{2}=0.39$. Happy and angry face processing was associated with enlarged EPN amplitudes compared to neutral stimuli, $F_{s_{(1,25)}}=37.91$ and 10.94, $p<0.001$ and 0.01, $\eta_{p}^{2}=0.60$ and 0.30. Further, the EPN was more pronounced for happy compared to angry faces, $F_{(1,25)}=5.05, p<0.05, \eta_{p}^{2}=0.17$. In addition, more pronounced negativities were observed over the left in contrast to the right hemisphere, $F_{(1,25)}=12.30, p<0.01, \eta_{p}^{2}=0.33$. No further interactions including Facial Expression, Task, and Relevance reached statistical significance, $F s<1.1$, ps $>0.33$, $\eta_{p}^{2}<0.05$

\section{Late positive potential}

Broadly distributed LPP were modulated by Task, $F_{(1,25)}=6.41$, $p<0.05, \eta_{p}^{2}=0.20$, and Facial Expression, $F_{(2,50)}=5.34, p=0.01$, $\eta_{p}^{2}=0.18$. Happy and angry faces elicited larger LPPs compared to neutral materials, $F s_{(1,25)}=11.46$ and 5.21, $p s<0.01$ and 0.05 , $\eta_{p}^{2}=0.31$ and 0.17 , although no difference was found between 

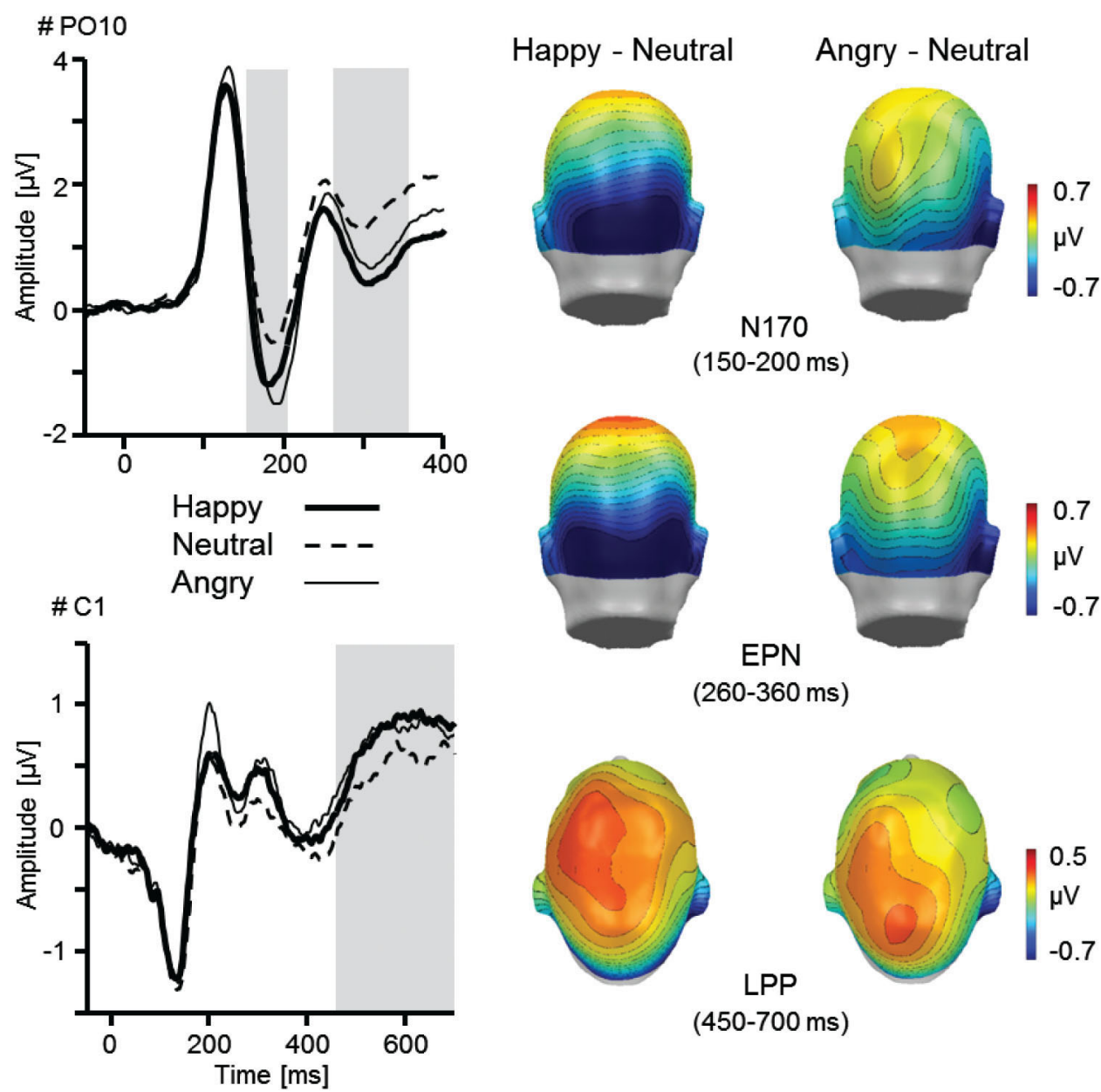

LPP

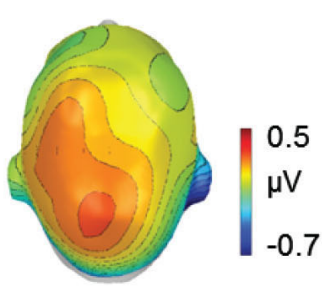

(450-700 ms)

FIGURE 2 | IIlustration of the main effect Facial Expression as revealed by the N170, EPN, and LPP component. ERP waveforms for an exemplary occipital (PO10) and central sensor (C1) for happy, neutral, and angry faces. Topographical difference maps (happy-neutral, angry-neutral) display the averaged time interval plotted on a back (N170: 150-200 ms EPN: 260-360 ms) and top view (LPP: 450-700 ms) of a model head Analyzed time windows are highlighted in gray (PO10: N170 and EPN; C1: LPP). happy and angry facial expressions, $F_{(1,25)}=0.23, p=0.64$, $\eta_{p}^{2}=0.01$. No differences were observed for instructed Relevance, $F_{(1,25)}=1.74, p=0.20, \eta_{p}^{2}=0.07$, and Laterality, $F_{(1,25)}<0.01$, $p=0.98, \eta_{p}^{2}<0.01$

Of particular interest, a significant interaction emerged for Facial Expression by Relevance, $F_{(2,50)}=3.6, p<0.05, \eta_{p}^{2}=0.12$. Further, a near-significant interaction was observed for Task by Relevance, $F_{(2,50)}=3.32, p=0.08, \eta_{p}^{2}=0.12$, but not for Facial Expression by Task, $F_{(2,50)}=2.18, p=0.14, \eta_{p}^{2}=0.08$, or the higher order interaction Facial Expression by Task by Relevance, $F_{(2,50)}=0.08, p=0.91, \eta_{p}^{2}<0.01$. To follow up these interactions, analyses were conducted separately for each experimental task (see Figure 4).

For the meet task, a significant main effect of Facial Expression was observed, $F_{(2,50)}=4.86, p<0.05, \eta_{p}^{2}=0.16$. Follow-up analyses revealed pronounced LPP amplitudes for happy faces, $F_{(1,25)}=9.33, p<0.01, \eta_{p}^{2}=0.27$, and marginally significant for angry compared to neutral facial expressions, $F_{(1,25)}=3.89$, $p=0.06, \eta_{p}^{2}=0.14$. No difference was observed between happy and angry facial expressions, $F_{(1,25)}=1.26, p=0.27, \eta_{p}^{2}=0.05$. Whereas, the interaction Facial Expression by Relevance did not reach significance, $F_{(2,50)}=1.83, p=0.18, \eta_{p}^{2}=0.07$, a near-significant main effect of Relevance was observed, $F_{(1,25)}=$ 3.85, $p=0.06, \eta_{p}^{2}=0.13$. Exploratory follow-up analyses testing relevant compared to non-relevant faces revealed enhanced LPP amplitudes for relevant happy faces, $F_{(1,25)}=4.12, p=0.05, \eta_{p}^{2}=$ 0.14 , but not for relevant neutral, $F_{(1,25)}=2.32, p=0.14, \eta_{p}^{2}=$ 0.09 , or angry faces, $F_{(1,25)}=0.02, p=0.91, \eta_{p}^{2}<0.01$.

In contrast, for the passive viewing task, only the main effect of Facial Expression reached marginal significance, $F_{(2,50)}=2.75, p=0.07, \eta_{p}^{2}=0.10$. Follow-up tests revealed pronounced LPP for angry faces, $F_{(1,25)}=4.47, p<0.05$, $\eta_{p}^{2}=0.15$, and marginally enhanced amplitudes for happy faces, $F_{(1,25)}=2.92, p=0.10, \eta_{p}^{2}=0.10$, compared to neutral facial 


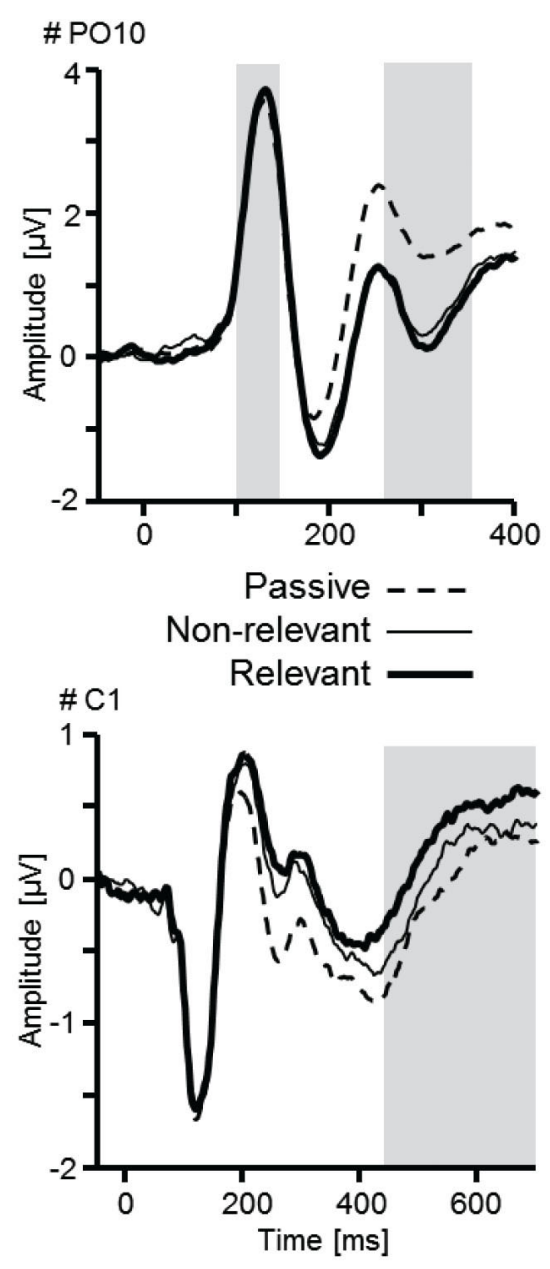

FIGURE 3 | Illustration of the main effects Task and Relevance as revealed by the P1, EPN, and LPP component. ERP waveforms for an exemplary occipital (PO10) and central sensor (C1) for relevant and non-relevant face stimuli, each compared to the passive viewing condition.
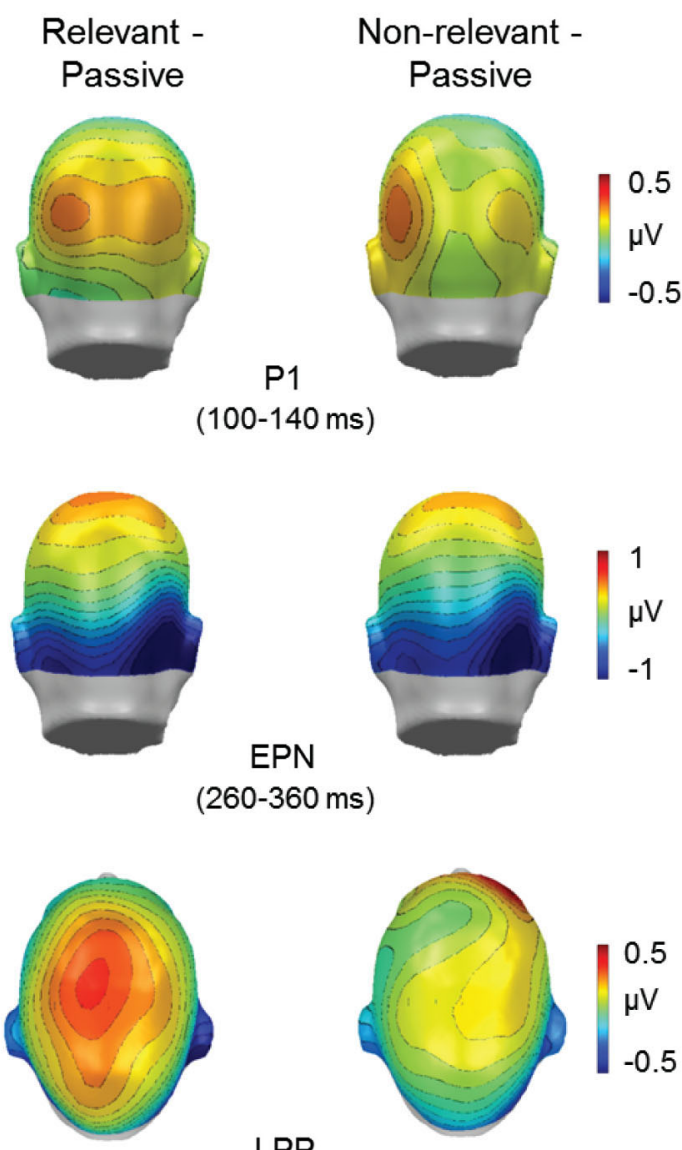

LPP

$(450-700 \mathrm{~ms})$

Topographical difference maps (relevant-passive, non-relevant-passive) display the averaged time interval plotted on the back of a model head (P1: 100-140 ms; EPN: 260-360 ms) and a top view (LPP: 450-700 ms). Analyzed time windows are highlighted in gray (PO10: P1 and EPN; C1: LPP). expressions. No difference was observed for happy and angry faces in the passive viewing task, $F_{(1,25)}=0.35, p=0.56$, $\eta_{p}^{2}=0.01$. Neither the main effect Relevance, $F_{(1,25)}<0.01$, $p=0.99, \eta_{p}^{2}<0.01$, and Laterality modulated LPP amplitudes, $F_{(1,25)}=0.03, p=0.86, \eta_{p}^{2}<0.01$, nor any interaction reached significance, $F s<1.86$, $p s>0.17, \eta_{p}^{2}<0.07$.

\section{DISCUSSION}

The present study examined the impact of instructed social relevance and emotional facial expression on face processing. The main finding was that the mere verbal instruction about social contingencies can modulate early and late face processing as indicated by enhanced P1, EPN, and LPP amplitudes. Importantly, event-related potential measures revealed that the interaction of social and emotional significance varied across visual processing stream. Whereas rather early components revealed independent main effects of facial expression and task instruction (P1, N170, and EPN), the LPP was augmented specifically for happy faces of future interaction partners. These results support the notion of joint impact of emotional and social information mediating face perception.

The anticipation of social interaction with another individual is of considerable value. In the present study, social relevance was manipulated by introducing two specific face actors as future interaction partners (meet task). Results indicate that this socioemotional context is associated with specific processing patterns as participants view face pictures. The first ERP component sensitive to both task instruction and emotional facial expression was the P1 component, which was enlarged for angry faces compared to happy and neutral facial expressions. Further, regardless of facial expression, enhanced P1 amplitudes were observed during meet compared to passive viewing task. Thus, several previous findings were replicated: enhanced P1 amplitudes in explicit attention tasks (Hillyard and Anllo-Vento, 1998; Pourtois et al., 2004) and implicit processing biases during self-relevant 

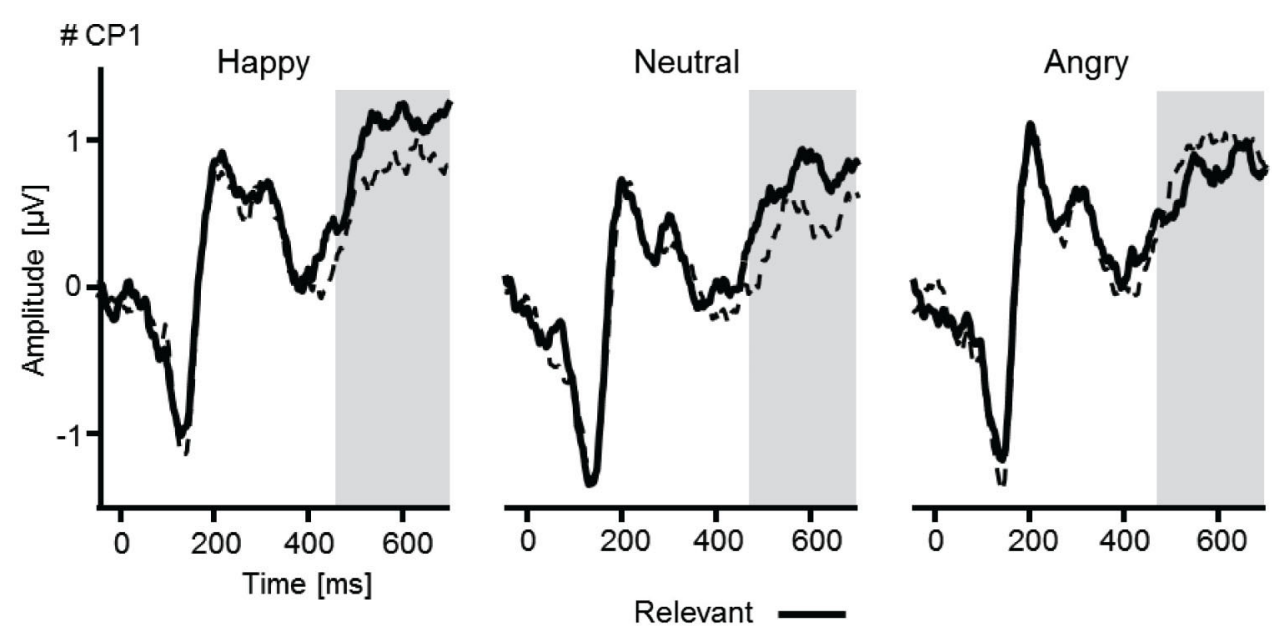

Non-relevant - - -

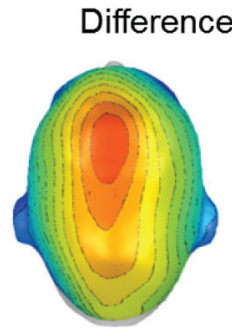

Happy

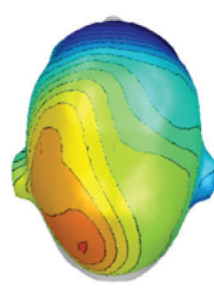

Neutral
0.7

$\mu \mathrm{V}$
FIGURE 4 | Illustration of Facial Expression as a function of Relevance as revealed by the LPP component. ERP waveforms for an exemplary

centro-parietal sensor (CP1) for happy, neutral, and angry faces when relevant or non-relevant. Topographical difference maps (relevant-non-relevant) display the averaged time interval (450-700 ms) plotted on a top view of a model head. Analyzed time window for the LPP are highlighted in gray. conditions (e.g., instructed threat or in specific phobia; Kolassa et al., 2006; Michalowski et al., 2009; Bublatzky and Schupp, 2012). Presumably based on intensified visual processing in the extrastriate cortex (Hillyard and Anllo-Vento, 1998; Pourtois et al., 2013), the present P1 effects may indicate enhanced vigilance during task conditions of high self-relevance.

Both N170 and EPN components varied as a function of emotional facial expression. As enhanced negativities have been found for both happy and angry compared to neutral faces, these findings suggest that selective face processing occurs as a function of stimulus arousal. Whereas the N170 has been mostly related to structural encoding of non-affective facial features (Sato et al., 2001; Eimer and Holmes, 2002) within occipitotemporal areas (e.g., STS; Itier and Taylor, 2004), the present data are in line with a growing body of literature showing that the N170 is subject to emotional modulation (Pizzagalli et al., 2002; Batty and Taylor, 2003; Rossignol et al., 2005) similar to the EPN component. Further, indicating the enhanced relevance of facial stimuli for (sub-) clinical populations with high levels of social anxiety, pronounced N170 and EPN amplitudes have been observed for angry facial expression (Kolassa and Miltner, 2006; Mühlberger et al., 2009; Wieser et al., 2010). Here, valence specific effects were observed in healthy participants, however, with more pronounced N170/EPN for happy facial expressions. One promising direction for future studies is to manipulate the implicit level of social relevance when examining interindividual differences in emotional face processing (e.g., familiar loved vs. unfamiliar faces displaying emotions; Guerra et al., 2012).

Regarding late positive potentials, face processing was modulated by both task- and emotional relevance. Similar to past research (Schupp et al., 2004), faces displaying angry expressions were associated with enhanced LPP amplitudes, however, this effect was similarly present for happy faces. Of particular interest, the social relevance manipulation revealed an interactive relationship with emotional facial expression. Whereas both happy and angry faces elicited an enhanced late parietal positivity compared to neutral stimuli, this effect was more pronounced when viewing potential interaction partner displaying happy facial expressions. A similar trend was observed for neutral, but not angry, faces of purported interaction partners compared to non-relevant faces. Thus, whereas emotional and social relevance independently modulated early ERP componentsindicating either a threat advantage (P1) or selective emotion processing (EPN)-later processing stage revealed specifically 
enhanced amplitudes for socially relevant happy faces (LPP). These findings appear in line with the evaluative space model of affective processing (Cacioppo and Berntson, 1994; Cacioppo et al., 1999). Depending on the level of activation, emotional input may provoke different processing and response gradients. For instance, at low activation levels, pleasant stimuli may exert a greater influence than unpleasant stimuli in guiding motivational tendencies (e.g., explorative behavior). Accordingly, in rather lowarousing experimental conditions, happy facial expression may be more efficient in activating the motivational approach system than angry faces fostering avoidance. This hypothesis could be tested with socially relevant faces presented under conditions of low and high arousal (e.g., threat-of-shock paradigm; Grillon and Charney, 2011; Bublatzky et al., 2010, 2013). Importantly, future research is needed to connect findings from the perceptual/attentional domain to the functional level, for instance, by testing approach/avoidance behavior (e.g., decision making; Pittig et al., 2014) to socially relevant happy/angry faces in social phobia (Wangelin et al., 2012).

Over and above the impact of implicit stimulus relevance (i.e., emotional facial expression), explicit instructions about social relevance in the meet task was associated with increased P1, EPN, and LPP amplitudes. These findings may complement recent research utilizing selective attention paradigms (Delorme et al., 2004; Pourtois et al., 2013). For instance, Schupp et al. (2007) observed pronounced EPN and enhanced late parietal positivities for target pictures of different semantic categories. Of particular interest, pictures displaying highly arousing content potentiated attention effects specifically during later processing stages (Schupp et al., 2007). In the present social anticipation task, the emotional facial features were no counting targets and actually rated as little arousing; however, a boost of emotion-focused attention was observable specifically for happy facial expression of purported interaction partner. Here, the reference to neural systems involved in various means of relevance processing based on bottom-up or top-down regulation may be informative (e.g., relevance based on task instruction, emotional, or social information; Schmitz and Johnson, 2007; Pourtois et al., 2013). For instance, paying attention to specific stimulus features modulates BOLD responses in the visual cortex (Kastner and Ungerleider, 2000), and for both emotional scenes and facial expressions a great overlap of neural activity has been demonstrated in the amygdala and medial prefrontal cortex (Sabatinelli et al., 2011); the latter being strongly involved in self-referential processing (Gusnard et al., 2001; Northoff et al., 2006; Olsson and Ochsner, 2008; Schwarz et al., 2013).

Several noteworthy aspects and alternative explanations of the present findings need to be acknowledged and should be addressed in future research. First, the critical test of the interaction between social relevance and facial expression was based on the processing of the same face stimuli that differed only in instructed social relevance. This approach has the advantage of ruling out potential effects due to physical differences, as apparent in comparing "social" vs. "non-social" stimuli, however, required that a fixed order of passive viewing task, followed by social meet instruction, was adopted. Thus, excessive stimulus repetitions may have reduced emotion or task effects.
However, similar to previous research (Codispoti et al., 2006; Schupp et al., 2006b), neither EPN nor LPP components revealed a reduction of selective emotion processing in the later task. On the contrary, the present LPP amplitudes were generally enhanced during social anticipation task. Furthermore, cognitive processes - such as working memory load or implicit emotion regulation-may have contributed to the absence of enhanced LPP to socially relevant angry faces. For instance, recent studies observed reduced LPP amplitudes to aversive stimuli under working memory load, suggesting that threat processing is contingent on available cognitive resources (MacNamara et al., 2011; Van Dillen and Derks, 2012). Alternatively, implicit emotion regulation may have reduced LPP amplitudes to aversive stimuli as shown in previous studies (Hajcak et al., 2010; Thiruchselvam et al., 2011). Here, future research may implement resource competition (e.g., by means of concurrent tasks or distractor stimuli) and active emotion regulation strategies. This could help clarify how social relevance affects emotional and cognitive processes in face perception.

The effects of selective attention elicited by either implicit emotional or explicitly instructed task relevance have been assessed in previous studies (Schupp et al., 2007; Pourtois et al., 2013). Extending this line of research, the present study utilized a novel approach to manipulate stimulus relevance by introducing specific face actors as future interaction partner. Social relevance was found to modulate face processing differently across visual processing stream. Whereas early ERP components revealed independent effects of social and emotional relevance (P1, N170, EPN), later processing stages were associated with specifically enhanced LPP for happy facial expressions when displayed by future interaction partner. Thus, social relevance may facilitate evaluative face processing according to socio-emotional settings (i.e., future interaction; Fischer and van Kleef, 2010).

\section{ACKNOWLEDGMENTS}

We are grateful to Susanne LaMura for her assistance in data collection. This work was supported, in part, by the "Struktur-und Innovationsfonds (SI-BW)" of the state of Baden-Wuerttemberg, Germany.

\section{REFERENCES}

Adolphs, R., and Spezio, M. (2006). Role of the amygdala in processing visual social stimuli. Prog. Brain Res. 156, 363-378. doi: 10.1016/s0079-6123(06) 56020-0

Alpers, G. W., Adolph, D., and Pauli, P. (2011). Emotional scenes and facial expressions elicit different psychophysiological responses. Int. J. Psychophysiol. 80, 173-181. doi: 10.1016/j.ijpsycho.2011.01.010

Alpers, G. W., and Gerdes, A. (2007). Here's looking at you: emotional faces predominate in binocular rivalry. Emotion 7, 495-506. doi: 10.1037/1528-3542. 7.3.495

Batty, M., and Taylor, M. J. (2003). Early processing of the six basic facial emotional expressions. Cogn. Brain Res. 17, 613-620. doi: 10.1016/s0926-6410(03) 00174-5

Bentin, S., Allison, T., Puce, A., Perez, E., and McCarthy, G. (1996). Electrophysiological studies of face perception in humans. J. Cogn. Neurosci. 8, 551-565. doi: 10.1162/jocn.1996.8.6.551

Bradley, M. M., Codispoti, M., Cuthbert, B. N., and Lang, P. J. (2001). Emotion and motivation I: defensive and appetitive reactions in picture processing. Emotion 1, 276-298. doi: 10.1037/1528-3542.1.3.276 
Bradley, M. M., and Lang, P. J. (1994). Measuring emotion: the self-assessment manikin and the semantic differential. J. Behav. Ther. Exp. Psychiatry 25, 49-59. doi: 10.1016/0005-7916(94)90063-9

Brothers, L. (2002). "The social brain: a project for integrating primate behavior and neurophysiology in a new domain," in Foundations in Social Neuroscience, ed J. T. Cacioppo (Cambridge: MIT Press), 367-384.

Bublatzky, F., Flaisch, T., Stockburger, J., Schmälzle, R., and Schupp, H. T. (2010). The interaction of anticipatory anxiety and emotional picture processing: an event-related brain potential study. Psychophysiology 47, 687-696. doi: 10.1111/j. 1469-8986.2010.00966.x

Bublatzky, F., Guerra, P., Pastor, M., Schupp, H. T., and Vila, J. (2013). Additive effects of threat-of-shock and picture valence on startle reflex modulation. PLoS One 8:e54003. doi: 10.1371/journal.pone.0054003

Bublatzky, F., and Schupp, H. T. (2012). Pictures cueing threat: brain dynamics in viewing explicitly instructed danger cues. Soc. Cogn. Affect. Neurosci. 7, 611-622. doi: 10.1093/scan/nsr032

Byrne, A., and Eysenck, M. W. (1995). Trait anxiety, anxious mood and threat detection. Cogn. Emot. 9, 549-562. doi: 10.1080/026999395084 08982

Cacioppo, J. T., and Berntson, G. G. (1994). Relationship between attitudes and evaluative space: a critical review, with emphasis on the separability of positive and negative substrates. Psychol. Bull. 115, 401-423. doi: 10.1037/0033-2909. 115.3.401

Cacioppo, J. T., Gardner, W. L., and Berntson, G. G. (1999). The affect system has parallel and integrative processing components: form follows function. J. Pers. Soc. Psychol. 76, 839-855. doi: 10.1037/0022-3514.76.5.839

Chao, L. L., Martin, A., and Haxby, J. V. (1999). Are face-responsive regions selective only for faces? Neuroreport 10, 2945-2950. doi: 10.1097/00001756199909290-00013

Codispoti, M., Ferrari, V., and Bradley, M. M. (2006). Repetitive picture processing: autonomic and cortical correlates. Brain Res. 1068, 213-220. doi: 10 1016/j.brainres.2005.11.009

Delorme, A., Rousselet, G. A., Macé, M. J.-M., and Fabre-Thorpe, M. (2004). Interaction of top-down and bottom-up processing in the fast visual analysis of natural scenes. Brain Res. Cogn. Brain Res. 19, 103-113. doi: 10.1016/j. cogbrainres.2003.11.010

Eimer, M., and Holmes, A. (2002). An ERP study on the time course of emotional face processing. Neuroreport 13, 427-431. doi: 10.1097/00001756-20020325000013

Ekman, P., and Friesen, W. V. (1975). Unmasking the Face. NJ: Prentice Hall Englewood Cliffs.

Fischer, A. H., and van Kleef, G. A. (2010). Where have all the people gone? A plea for including social interaction in emotion research. Emot. Rev. 2, 208-211. doi: $10.1177 / 1754073910361980$

Flaisch, T., Junghöfer, M., Bradley, M. M., Schupp, H. T., and Lang, P. J. (2008). Rapid picture processing: affective primes and targets. Psychophysiology 45, 110. doi: 10.1111/j.1469-8986.2007.00600.x

Flaisch, T., Schupp, H. T., Renner, B., and Junghöfer, M. (2009). Neural systems of visual attention responding to emotional gestures. Neuroimage 45, 1339-1346. doi: 10.1016/j.neuroimage.2008.12.073

Grillon, C., and Charney, D. R. (2011). In the face of fear: anxiety sensitizes defensive responses to fearful faces. Psychophysiology 48, 1745-1752. doi: 10 . 1111/j.1469-8986.2011.01268.x

Guerra, P., Vico, C., Campagnoli, R. R., Sánchez, A., Anllo-Vento, L., and Vila, J. (2012). Affective processing of loved familiar faces: integrating central and peripheral electrophysiological measures. Int. J. Psychophysiol. 85, 79-87. doi: 10. 1016/j.ijpsycho.2011.06.004

Gusnard, D. A., Akbudak, E., Shulman, G. L., and Raichle, M. E. (2001). Medial prefrontal cortex and self-referential mental activity: relation to a default mode of brain function. Proc. Natl. Acad. Sci. U S A 98, 4259-4264. doi: 10.1073/pnas. 071043098

Hajcak, G., MacNamara, A., and Olvet, D. M. (2010). Event-related potentials, emotion and emotion regulation: an integrative review. Dev. Neuropsychol. 35 , 129-155. doi: 10.1080/87565640903526504

Hamm, A. O., Schupp, H. T., and Weike, A. I. (2003). "Motivational organization of emotions: autonomic changes, cortical responses and reflex modulation," in Handbook of Affective Sciences, eds R. J. Davidson, K. R. Scherer and H. H. Goldsmith (Oxford: Oxford University Press), 187-211.
Haxby, J. V., Hoffman, E. A., and Gobbini, M. I. (2002). Human neural systems for face recognition and social communication. Biol. Psychiatry 51, 59-67. doi: 10. 1016/s0006-3223(01)01330-0

Hillyard, S. A., and Anllo-Vento, L. (1998). Event-related brain potentials in the study of visual selective attention. Proc. Natl. Acad. Sci. U S A 95, 781-787. doi: 10.1073/pnas.95.3.781

Holmes, A., Nielsen, M. K., and Green, S. (2008). Effects of anxiety on the processing of fearful and happy faces: an event-related potential study. Biol. Psychol. 77, 159-173. doi: 10.1016/j.biopsycho.2007.10.003

Holmes, A., Vuilleumier, P., and Eimer, M. (2003). The processing of emotional facial expression is gated by spatial attention: evidence from event-related brain potentials. Brain Res. Cogn. Brain Res. 16, 174-184. doi: 10.1016/s0926-6410(02) 00268-9

Itier, R. J., Latinus, M., and Taylor, M. J. (2006). Face, eye and object early processing: what is the face specificity? Neuroimage 29, 667-676. doi: 10.1016/j. neuroimage.2005.07.041

Itier, R. J., and Taylor, M. J. (2002). Inversion and contrast polarity reversal affect both encoding and recognition processes of unfamiliar faces: a repetition study using ERPs. Neuroimage 15, 353-372. doi: 10.1006/nimg.2001.0982

Itier, R. J., and Taylor, M. J. (2004). Source analysis of the N170 to faces and objects. Neuroreport 15, 1261-1265. doi: 10.1097/01.wnr.0000127827.73576.d8

Jacques, C., and Rossion, B. (2007). Electrophysiological evidence for temporal dissociation between spatial attention and sensory competition during human face processing. Cereb. Cortex 17, 1055-1065. doi: 10.1093/cercor/bhl015

Junghöfer, M., Elbert, T., Tucker, D. M., and Rockstroh, B. (2000). Statistical control of artifacts in dense array EEG/MEG studies. Psychophysiology 37, 523-532. doi: 10.1111/1469-8986.3740523

Junghöfer, M., Sabatinelli, D., Bradley, M. M., Schupp, H. T., Elbert, T. R., and Lang, P. J. (2006). Fleeting images: rapid affect discrimination in the visual cortex. Neuroreport 17, 225-229. doi: 10.1097/01.wnr.0000198437.59 883.bb

Kanwisher, N., McDermott, L. G., and Chun, M. M. (1997). The fusiform face area: a module in human extrastriate cortex specialized for face perception. J. Neurosci. 17, 4302-4311.

Kastner, S., and Ungerleider, L. G. (2000). Mechanisms of visual attention in the human cortex. Annu. Rev. Neurosci. 23, 315-341. doi: 10.1146/annurev.neuro. 23.1.315

Kaufmann, J. M., Schweinberger, S. R., and Burton, A. M. (2008). N250 ERP correlates of the acquisition of face representation across different images. J. Cogn. Neurosci. 21, 625-641. doi: 10.1162/jocn.2009.21080

Keltner, D., and Kring, A. M. (1998). Emotion, social function and psychopathology. Rev. Gen. Psychol. 2, 320-342. doi: 10.1037/1089-2680.2. 3.320

Kissler, J., Herbert, C., Peyk, P., and Junghöfer, M. (2007). Buzzwords: early cortical responses to emotional words during reading. Psychol. Sci. 18, 475-480. doi: 10. 1111/j.1467-9280.2007.01924.x

Kolassa, I.-T., Musial, F., Kolassa, S., and Miltner, W. H. R. (2006). Event-related potentials when identifying or color-naming threatening schematic stimuli in spider phobic and non-phobic individuals. BMC Psychiatry 6:38. doi: 10 1186/1471-244X-6-38

Kolassa, I.-T., and Miltner, W. H. R. (2006). Psychophysiological correlates of face processing in social phobia. Brain Res. 1118, 130-141. doi: 10.1016/j.brainres. 2006.08.019

Liddell, B. J., Williams, L. M., Rathjen, J., Shevrin, H., and Gordon, E. (2004). A temporal dissociation of subliminal versus supraliminal fear perception: an event-related potential study. J. Cogn. Neurosci. 16, 479-486. doi: 10. $1162 / 089892904322926809$

Lundqvist, D., Flykt, A., and Öhman, A. (1998). The Karolinska Directed Emotional Faces - KDEF, CD ROM from Department of Clinical Neuroscience, Psychology section, Karolinska Institutet, ISBN 630-7164-9.

MacNamara, A., Ferri, J., and Hajcak, G. (2011). Working memory load reduces the late positive potential and this effect is attenuated with increasing anxiety. Cogn. Affect. Behav. Neurosci. 11, 321-331. doi: 10.3758/s13415-0110036-z

Michalowski, J. M., Melzig, C. A., Weike, A. I., Stockburger, J., Schupp, H. T., and Hamm, A. O. (2009). Brain dynamics in spider-phobic individuals exposed to phobia-relevant and other emotional stimuli. Emotion 9, 306-315. doi: 10. 1037/a0015550 
Moser, J. S., Huppert, J. D., Duval, E., and Simons, R. F. (2008). Face processing biases in social anxiety: an electrophysiological study. Biol. Psychol. 78, 93-103. doi: 10.1016/j.biopsycho.2008.01.005

Mühlberger, A., Wieser, M. J., Hermann, M. J., Weyers, P., Tröger, C., and Pauli, P. (2009). Early cortical processing of natural and artificial emotional faces differs between lower and higher socially anxious persons. J. Neural Transm. 116, 735746. doi: 10.1007/s00702-008-0108-6

Norris, C. J., Chen, E. E., Zhu, D. C., Small, S. L., and Cacioppo, J. T. (2004). The interaction of social and emotional processes in the brain. J. Cogn. Neurosci. 16, 1818-1829. doi: 10.1162/0898929042947847

Northoff, G., Heinzel, A., de Greck, M., Bermpohl, F., Dobrowolny, H., and Panksepp, J. (2006). Self-referential processing in our brain-A meta-analysis of imaging studies on self. Neuroimage 31, 440-457. doi: 10.1016/j.neuroimage. 2005.12.002

Öhman, A. (1986). Face the beast and fear the face: animal and social fears as prototypes for evolutionary analyses of emotion. Psychophysiology 23, 123-145. doi: 10.1111/j.1469-8986.1986.tb00608.x

Öhman, A., Lundqvist, D., and Esteves, F. (2001). The face in the crowd revisited: a threat advantage with schematic stimuli. J. Pers. Soc. Psychol. 80, 381-396. doi: 10.1037//0022-3514.80.3.381

Olsson, A., and Ochsner, K. N. (2008). The role of social cognition in emotion. Trends Cogn. Sci. 12, 65-71. doi: 10.1016/j.tics.2007.11.010

Peyk, P., De Cesaire, A., and Junghöfer, M. (2011). Electromagnetoencephalography software: overview and integration with other EEG/MEG toolboxes. Comput. Intell. Neurosci. 2011:861705. doi: 10.1155/2011/861705

Pittig, A., Schulz, A. R., Craske, M. G., and Alpers, G. W. (2014). Acquisition of behavioral avoidance: task-irrelevant conditioned stimuli trigger costly decisions. J. Abnorm. Psychol. 123, 314-329. doi: 10.1037/a0036136

Pizzagalli, D. A., Lehmann, D., Hendrick, A. M., Regard, M., Pascual-Marqui, R. D., and Davidson, R. J. (2002). Affective judgements of faces modulate early activity within the fusiform gyri. Neuroimage 16, 663-677. doi: 10.1006/nimg.2002.1126

Pourtois, G., Grandjean, D., Sander, D., and Vuilleumier, P. (2004). Electrophysiological correlates of rapid spatial orienting towards fearful faces. Cereb. Cortex 14, 619-633. doi: 10.1093/cercor/bhh023

Pourtois, G., Schettino, A., and Vuilleumier, P. (2013). Brain mechanisms for emotional influences on perception and attention: what is magic and what is not. Biol. Psychol. 92, 492-512. doi: 10.1016/j.biopsycho.2012.02.007

Rossignol, M., Philippot, P., Douilliez, C., Crommelinck, M., and Campanella, S. (2005). The perception of fearful and happy facial expression is modulated by anxiety: an event-related potential study. Neurosci. Lett. 377, 115-120. doi: 10. 1016/j.neulet.2004.11.091

Sabatinelli, D., Fortune, E. E., Li, Q., Siddiqui, A., Krafft, C., Oliver, W. T., et al. (2011). Emotional perception: meta-analyses of face and natural scene processing. Neuroimage 54, 2524-2533. doi: 10.1016/j.neuroimage.2010.10.011

Sato, W., Kochiyama, T., Yoshikawa, S., and Matsumura, M. (2001). Emotional expression boosts early visual processing of the face: ERP recording and its decomposition by independent component analysis. Neuroreport 12, 709-714. doi: 10.1097/00001756-200103260-00019

Schacht, A., and Sommer, W. (2009). Emotions in word and face processing: early and late cortical responses. Brain Cogn. 69, 538-550. doi: 10.1016/j.bandc.2008. 11.005

Schmitz, T. W., and Johnson, S. C. (2007). Relevance to self: a brief review and framework of neural systems underlying appraisal. Neurosci. Biobehav. Rev. 31, 585-596. doi: 10.1016/j.neubiorev.2006.12.003

Schupp, H. T., Flaisch, T., Stockburger, J., and Junghöfer, M. (2006a). Emotion and attention: event-related brain potential studies. Prog. Brain Res. 156, 31-51. doi: 10.1016/s0079-6123(06)56002-9

Schupp, H. T., Öhman, A., Junghöfer, M., Weike, A. I., Stockburger, J., and Hamm, A. O. (2004). The facilitated processing of threatening faces: an ERP analysis. Emotion 4, 189-200. doi: 10.1037/1528-3542.4.2.189

Schupp, H. T., Stockburger, J., Codispoti, M., Junghöfer, M., Weike, A. I., and Hamm, A. O. (2006b). Stimulus novelty and emotion perception: the near absence of habituation in the visual cortex. Neuroreport 17, 365-369. doi: 10 . 1097/01.wnr.0000203355.88061.c6

Schupp, H. T., Stockburger, J., Codispoti, M., Junghöfer, M., Weike, A. I., and Hamm, A. O. (2007). Selective visual attention to emotion. J. Neurosci. 27, 10821089. doi: 10.1523/JNEUROSCI.3223-06.2007

Schwarz, K. A., Wieser, M. J., Gerdes, A. B. M., Mühlberger, A., and Pauli, P. (2013). Why are you looking like that? How the context influences evaluation and processing of human faces. Soc. Cogn. Affect. Neurosci. 8, 438-445. doi: 10. 1093/scan/nss013

Schweinberger, S. R., Pickering, E. C., Burton, A. M., and Kaufmann, J. M. (2002). Human brain potential correlates of repetition priming in face and name recognition. Neuropsychologia 40, 2057-2073. doi: 10.1016/s0028-3932(02) 00050-7

Sewell, C., Palermo, R., Atkinson, C., and McArthur, G. (2008). Anxiety and the neural processing of threat in faces. Neuroreport 19, 1339-1343. doi: 10 . 1097/WNR.0b013e32830baadf

Thiruchselvam, R., Blechert, J., Sheppes, G., Rydstrom, A., and Gross, J. J. (2011). The temporal dynamics of emotion regulation: an EEG study of distraction and reappraisal. Biol. Psychol. 87, 84-92. doi: 10.1016/j.biopsycho.2011.02.009

Tooby, J., and Cosmides, L. (1992). "Psychological foundations of culture," in The Adapted Mind: Evolutionary Psychology and the Generation of Culture, eds J. H. Barkow, L. Cosmides and J. Tooby (New York: Oxford University Press), 19-136.

Van Dillen, L. F., and Derks, B. (2012). Working memory load reduces facilitated processing of threatening faces: an ERP study. Emotion 12, 1340-1349. doi: 10. 1037/a0028624

Vuilleumier, P., and Pourtois, G. (2007). Distributed and interactive brain mechanisms during emotion face perception: evidence from functional neuroimaging. Neuropsychologia 45, 174-194. doi: 10.1016/j.neuropsychologia.2006.06.003

Wangelin, B. C., Bradley, M. M., Kastner, A., and Lang, P. J. (2012). Affective engagement for facial expressions and emotional scenes: the influence of social anxiety. Biol. Psychol. 91, 103-110. doi: 10.1016/j.biopsycho.2012.05.002

Whalen, P. J., Shin, L. M., McInerney, S. C., Fischer, H., Wright, C. I., and Rauch, S. L. (2001). A functional MRI study of human amygdala responses to facial expressions of fear versus anger. Emotion 1, 70-83. doi: 10.1037//1528-35 42.1.1.70

Wieser, M. J., Pauli, P., Reicherts, P., and Mühlberger, A. (2010). Dont look at me in anger! Enhanced processing of angry faces in anticipation of public speaking. Psychophysiology 47, 271-280. doi: 10.1111/j.1469-8986.2009.00938.x

Williams, M. A., Morris, A. P., McGlone, F., Abbott, D. F., and Mattingley, J. B. (2004). Amygdala responses to fearful and happy facial expressions under conditions of binocular suppression. J. Neurosci. 24, 2898-2904. doi: 10 . 1523/jneurosci.4977-03.2004

Williams, L. M., Palmer, D., Liddell, B. J., Song, L., and Gordon, E. (2006). The when and where of perceiving signals of threat versus non-threat. Neuroimage 31, 458-467. doi: 10.1016/j.neuroimage.2005.12.009

Conflict of Interest Statement: The authors declare that the research was conducted in the absence of any commercial or financial relationships that could be construed as a potential conflict of interest.

Received: 30 April 2014; accepted: 17 June 2014; published online: 16 July 2014.

Citation: Bublatzky F, Gerdes ABM, White AJ, Riemer M and Alpers GW (2014) Social and emotional relevance in face processing: happy faces of future interaction partners enhance the late positive potential. Front. Hum. Neurosci. 8:493. doi: 10.3389/fnhum.2014.00493

This article was submitted to the journal Frontiers in Human Neuroscience.

Copyright (C) 2014 Bublatzky, Gerdes, White, Riemer and Alpers. This is an open-access article distributed under the terms of the Creative Commons Attribution License (CC $B Y)$. The use, distribution or reproduction in other forums is permitted, provided the original author(s) or licensor are credited and that the original publication in this journal is cited, in accordance with accepted academic practice. No use, distribution or reproduction is permitted which does not comply with these terms. 